W. H. Cliff and A. W. Wright

Advan Physiol Educ 270:19-28, 1996.

You might find this additional information useful...

Medline items on this article's topics can be found at http://highwire.stanford.edu/lists/artbytopic.dtl

on the following topics:

Communication .. Case Studies

Education .. Textbooks

Physiology .. Humans

Additional material and information about Advances in Physiology Education can be found at: http://www.the-aps.org/publications/advan

This information is current as of October 23, 2007.

Advances in Physiology Education is dedicated to the improvement of teaching and learning physiology, both in specialized courses and in the broader context of general biology education. It is published four times a year in March, June, September and December by the American Physiological Society, 9650 Rockville Pike, Bethesda MD 20814-3991. Copyright (C) 2005 by the American Physiological Society. ISSN: 1043-4046, ESSN: 1522-1229. Visit our website at http://www.the-aps.org/. 


$\begin{array}{llllllllllllllllllll}\text { I } & N & N & O & V & A & T & \text { I } & O & N & S & A & N & D & \text { I } & D & E & A & S\end{array}$

\title{
DIRECTED CASE STUDY METHOD FOR TEACHING HUMAN ANATOMY AND PHYSIOLOGY
}

\author{
William H. Cliff and Ann W. Wright \\ Department of Biology, Niagara University, Niagara, New York 14109
}

\begin{abstract}
A mastery of human anatomy and physiology requires a familiarity with a vast number of details about the human body. A directed method of case analysis is described that helps students deepen and solidify their understanding of anatomical and physiological facts, concepts, and principles. The successful case had four distinctive features as follows: clear learning objectives, a concise and informative scenario, straightforward and didactic questions, and an emphasis on information readily available to the student. A directed case study is presented, and its salient features are described. A procedure for integrating case analyses into an undergraduate anatomy and physiology course is outlined. Student response to this type of case study suggests that this method improves the ease of learning, the depth of learning, and an appreciation of the relevance of and a curiosity about anatomy and physiology. The addition of case analyses to a two-semester integrated course in anatomy and physiology was also associated with an improvement in exam performance. The regular use of directed case analysis is a valuable addition to the traditional methods of lecture, textbook reading, and laboratory for the teaching of human anatomy and physiology.
\end{abstract}

AM. J. PHYSIOL. 270 (ADV. PHYSIOL. EDUC. 15): S19-S28, 1996.

Key words: case studies; teaching undergraduate students; evaluation

A "case" for the case method in buman anatomy and physiology. Human anatomy and physiology is undeniably a content-rich systematic course of study. Our students must know when the atrioventricular valves close during the heart beat, what all of the cranial nerves (and their functions) are, where the spleen is, how muscles contract, and many other details about the human body. A mastery of the subject has been recognized as the ability to correctly recall a substantial fraction of this massive corpus of facts. The teaching of anatomy and physiology has focused on improving the student's capability to recall the pertinent ones. Lectures, textbooks, and to some extent the laboratories are designed to make this process easier.

Nevertheless, there are a number of reasons why a case analysis approach to teaching anatomy and physiology can be a useful adjunct to the traditional lecture-textbook-laboratory method of instruction in an undergraduate course in human anatomy and physiology.

1) Human anatomy and physiology is a foundational course for advanced topics in the basic or clinical sciences that put increasing emphasis on problem solving. Why not help students develop the necessary synthetic, analytic, and diagnostic thinking skills early in their program of study by introducing case analysis?

2) The teaching of human anatomy and physiology often introduces subjects (description of disease processes, clinical procedures and issues of health and fitness) explored or emphasized in greater detail in more advanced clinical courses. A case that deals with disease or pathology often deepens the student's 
understanding of the normal human anatomy and physiology, proves immediate real-life relevance, and helps develop an appreciation that the mastery of human anatomy and physiology is essential for achieving the clinical expertise of a competent health professional.

3) Mcre accumulation of a massive body of facts can never be the sole goal for this or any similar type of course. Students have become truly knowledgeable if they can correctly apply the facts that they have learned about the human body to the solution of relevant real-world problems.

The potcntial that case analysis holds in the teaching of human anatomy and physiology has been recognized in some introductory anatomy and physiology textbooks. Recent publications of Marieb (4), Martini (6) and Van Wynsberghe et al. (10) include case studies in the teaching materials. Some texts provide brief case scenarios at the end of each chapter (see Refs. 4 and 6). Others provide more extensive cases at the end of the major sections of the text (10). The Student's Application book for Martini's third edition textbook (7) is a good illustration of how cases are being integrated into the textbook supplements. Independent case study texts also exist. Van Wynsberghe and Cooley's "Case Histories in Human Physiology" (9) or Berne and Levy's "Case Studies in Physiology" (1) are particularly well-written examples. The latter was prepared for medical students.

These case approaches provide a strong clinical correlate to the basic science taught in the course. Nevertheless, much of this material does not appear to have been specifically written to reinforce or deepen the student's understanding of the anatomy or physiology. Instead, the cases serve primarily to illustrate disease states, give real-world relevance, and provide motivation for learning. The strength of these approaches is the development of clinical problem-solving skills by the student. The case method described in this paper retains the true-to-life clinical relevance found in contemporary texts. However, it has the primary goal of enhancing the student's understanding of the key concepts and processes of human anatomy and physiology.

The distinctions of a directed case study approach. Because the intent of the case method described here is the mastery of ideas and processes, the cases are content driven. They are built around specific learning objectives. Furthermore, the cases are highly directed. The student is asked a series of specific questions concerning the case. By answering these questions, a student is forced to review, relearn, and apply information that he or she has already gained from the text or from the lecture. Commonly, our cases resemble the decision or dilemma case (2) in which a scenario is presented, and a student must assemble the relevant information, identify key concepts, and make informed assessments to solve a problem. The goal is to obtain a deeper "working" knowledge of the material rather than simply captivate attention with interesting clinical situations.

Being directed, the cases are not open-ended. There is a single specific answer expected for nearly all of the questions. Open-ended cases foster intellectual development as students consider opposing or alternate possibilities in their approach to the solution of a problem. Nevertheless, such a format can also cause confusion and cognitive frustration if students do not have the prerequisite intellectual maturity to handle the open-endedness of this method. We have found the use of directed case analysis to be necessary for lower-division students. They still need guidance in applying their knowledge to real-world problems. Because our primary goal is to strengthen our student's grasp of the factual and conceptual aspects of anatomy and physiology and secondarily to develop greater critical thinking skills, we have found the directed case to be the most suitable approach.

\section{PROCEDURE/METHOD}

Directed case approach to teaching human anatomy and physiology. Four distinguishing features characterized our directed case approach as follows: 1) defined, inclusive learning objectives; 2) an informative, engaging case scenario; 3 ) pertinent, didactic questions; and 4 ) information needed to answer the case questions is readily available to students.

We found a well-prepared series of learning objectives to be invaluable in the construction of an instructive case. Typically, we began with a list of anatomical and physiological principles that are to be illustrated by the case (i.e., "three phases of the control of gastric secretion"). Then we defined the particular aspects of 
each principle that were to be emphasized ("the interaction between neural and hormonal mechanisms in the control of each phase of secretion"). Finally, we considered what idea or fact the student should know after completing the case analysis ("understand the relations between the neural and hormonal control of gastric secretion"). In our experience, the best case objectives should not be so narrow as to exclude key principles or essential concepts. On the other hand, the objectives should not be so broad as to make the case too diffuse or unrealistic.

The case scenario could be a clinical case history or a situation of medical importance, but this is not required. It could be a scientific or real-world concern related to human anatomy and physiology. For example, to reinforce skeletal anatomy, one could construct a puzzle about the peculiar shapes of human bones found at an ancient burial site. In our experience, the best scenarios were short (5-10 sentences at most), informative, and clearly written, giving all relevant information without unnecessary or obscuring detail. They were not too complex, difficult, or involved. Nor were they too simple, easy, or brief. They were interesting-sparking a reader's curiosity. They could be entertaining-but not to the detriment of their instructional value. Throughout the development of the scenario, carcful considcration was given to the relevant principles of anatomy and physiology that are to be emphasized and how the scenario illustrated them.

We also constructed the questions with the underlying principles of anatomy and physiology in mind. The questions needed to be relevant; they must direct the reader to apply his/her anatomical and physiological knowledge to the case at hand. The questions needed to be instructive; their intent is to force the reader to gain a greater understanding of human anatomy and physiology (What specific types of cells, tissues, organs, or organ systems are involved, i.e., what is the normal anatomy? How do these structures work normally, i.e., what is the normal physiology?). Because we wanted to place emphasis on the basic sciences, we decided that most of the questions should not have a clinical focus (i.e., What is the disease? What is its prognosis? What is the treatment?).

We believe that students should be able to successfully answer the case questions with information that can be obtained in the lectures, the course textbook, and supplements. This approach focuses student attention on the lectures and the textbook and discourages inefficient searches through advanced source materials. Occasionally, we directed our students to specialized readings but only if it enhanced their understanding of the subject. For some cases, it was not unreasonable to expect students to create answers to all of the questions from information found solely in the textbook. In doing so, case work enabled students to learn a wider scope of material than could be presented in the lecture.

We wrote case studics for each of the major systems (i.e., muscular, nervous, cardiovascular) covered in a typical two-semester integrated anatomy and physiology course. All of the cases drew upon concurrent knowledge of the anatomy and physiology of each major body system. Tables of data or figures for drawing graphs were provided. Diagrams were given for exercises involving labeling or identifying anatomical structures. Research literature or short generalinterest articles were made available on reserve in the library. A detailed handout giving suggestions for successfully answering the case study questions was given out at the beginning of the semester (see APPENDIX 1).

We found that a short focused scenario combined with 10-15 directed questions provided sufficient depth of analysis without requiring excessive time or effort by the students. Because students would typically complete six to eight case analyses per semester, a requirement for short and concise cases was appreciated by students and instructors alike.

\section{Representative case in buman anatomy and physiol-} $o g y$. A representative case in human anatomy and physiology is given in APPFNDIX II. This case concerns the immune system and its role in the allergic response.

Notice that the first five learning objectives are limited to the physiology of an allergic response. Nevertheless, embedded with these objectives is the implicit understanding of other important immunological concepts (i.e., the role of macrophages, B cells, and antibodies; the process of antigen sensitization; and the physiology of inflammation). Furthermore, objec- 
tives 6 and 7 are distinctly integrative goals in which the student must consider how alterations in immune cell function can lead to changes in the cardiovascular system, the respiratory system, and body fluid balance. Thus, although the case was limited to the allergic response, it covered a broader spectrum of topics in immunology and cardiovascular, respiratory, and body fluid physiology.

The case is concise. The clinical symptoms of the allergic reaction are summarized without embellishment. The events leading up to the main character's allergic reaction are outlined succinctly. The events are realistic even though the plot itself is fictitious. Many students have heard of similar real-life occurrences. The man's situation is painfully humorous. I'he realism and the humor are designed to catch the student's attention and maintain his or her interest. The specific cause or explanation for the man's condition is not given, and none of the facts are obscured. The student must decide which of the man's actions led to his predicament and analyze the reasons why.

The questions require a balance of fact recall, logical explanation, and synthetic analysis. From the information provided in the scenario and available in the textbook, the student is expected to diagnosis the man's pathological state. The students are asked to review the cellular steps involved in antigen sensitization and arrange them in proper sequence. The students must identify the salient features of inflammation and attribute them to the actions of specific cells and tissues. The student is expected to understand the physiological rationale for the treatment given. In all of these activities, a student develops a synthetic or analytic outlook to the problem instead of merely reinforcing his or her talent for fact recall. Nevertheless, most questions do indecd direct the student's attention to the facts and concepts of normal anatomy and physiology. This represents an opportunity for the student to review, reinforce, and gain a greater comprehension of the subject (i.e., the function of the immune system) instead of learning new information that may be tangential to the course (i.e., the treatment of allergic reactions). Moreover, some questions are integrative, requiring the student to associate the actions of one body system (i.e., release of inflammatory mediators by immune cells) with the responses of other systems (i.e., the effects on vasodilation and capillary permeability and the state of the cardiovascular system).

That nearly all of the answers to the case have citations to the text or supplements illustrates that the student can find facts, draw conclusions, or make inferences from information given in the text, lecture notes, or supplemental readings. Sometimes, this requirement might limit the scope of the case or restrict its logical development. However, given the wealth of information in modern texts in anatomy and physiology, we did not find that this restriction hampered our ability to construct informative cases or to use them effectively.

Use of directed case analysis in a course in buman anatomy and physiology. We used the following procedure to integrate a regular program of case analyses into our two-semester anatomy and physiology course.

1) The students received a case study at the beginning of each of the lecture series on each major system of the body. Students handed in their answers during the class period immediately after the last lecture in the series. The answers to the case study were reviewed at this time. In a typical two-semester sequence, this meant that the students had the case studies for at least a week before they were due. This schedule was designed so that students could anticipate gaining insight about the case as they learned about the body system in the lecture or from reading the textbook.

2) The students completed the case analysis outside of class. They werc frec to work together in groups. Many used their lab partners as co-workers on the cases. Students were told that information provided in class or found in the textbook should be sufficient to answer the questions. However, they were free to use any sources of information.

3) The students were required to turn in their written answers on or before the day of the case study review. Severe penalties were assessed for late answers, since the answers were fully discussed in class on the day that they were due.

4) After the written answers were handed in, each question was reviewed in sequence by having indi- 
vidual students read their answers to the class. The instructor initiated a give-and-take class discussion about each answer, during which time the instructor had the opportunity to make additional comments about the case, and the students could raise specific concerns about the questions and/or answers. In such a fashion, the case study was completely reviewed in class. Sometimes, a formal quiz was administered at the beginning of class to assess the student's individual understanding of the material.

5) The written answers were graded and returned to the students. An answer key was posted in the student laboratory.

Providing a case study for each of the major body systems covered in the course made the cases a practical study aid and promoted immediate reinforcement of the lecture material as it was presented in class. Our students also used their corrected case analyses in preparation for their exams.

Students were encouraged to work on the cases in groups, and no penalties were imposed for case answers jointly submitted by the students who worked together. In this way, the case approach was a useful extension of the group learning methods that we have used in the laboratory portion of the course. Individual comprehension of the subjects covered in the case studies was assessed by the scores on case-related questions on quizizes and/or exams.

We had no reservations about scheduling an entire class period to review each case (6-8 class periods/ semester). In nearly every instance, we used the full 50 min of class time to discuss the correct answers; clarify misunderstandings about the case scenario, questions, and answers; and review the related ideas and principles of anatomy and physiology. This time provided us with a valuable opportunity to reinforce and reemphasize subject material presented previously in the lecture. Furthermore, these review sessions also gave us a chance to improve our assessment of student learning.

\section{RESULTS}

Student response. An anonymous survey was made of the entire class of human anatomy and physiology students at the end of the first year after the adoption of the case analyses. Students were asked to indicate the extent of their agreement or disagreement with each statement. Responses were received from 66 of the 68 students who completed the two-semester course sequence. Greater than $65 \%$ of the class agreed or agreed somewhat that their work on the case studics 1) made it easier to learn anatomy and physiology $(74 \%), 2)$ helped to deepen or solidify their understanding of anatomy and physiology (70\%), 3) made them appreciate the relevance of learning anatomy and physiology (65\%), 4) made them curious to learn more advanced concepts of anatomy and physiology (67\%), and 5) were a useful way of learning anatomy and physiology (71\%). These percentages show that a substantial majority of students found case studies to be beneficial to their learning and appreciation of anatomy and physiology.

Considering the extra effort required by the students, it was not unexpected that the overwhelming majority found the case studies to be "challenging" (91\%). However, we were surprised that only $41 \%$ disagreed or disagreed somewhat with the statement that solving the case studies was "fun." We had expected that a large majority of the students would disagree, since the cases involved substantial out-of-class work without an explicit guarantee of success. Indeed, we were pleased that a notable percentage of students $(27 \%)$ agreed somewhat with the statement that solving the case studies was fun. This shows that, although the cases made our students work hard (i.e., were challenging), most of our students did not find the work unpleasant.

Student performance. By the objective measure of test scores, the addition of case study analyses was associated with an improvement in the student's performance on in-class exams. See Table 1 for the exam scores for our two-semester course in anatomy and physiology before and after the incorporation of case study analysis. 'The mean increase in exam scores was 7 out of $100(P=0.002 ; t$-test, unpaired comparison, $n=6$ exams). There was no substantial change in content or level of difficulty of the course between these two years.

Some of the difference could be attributed to year-toyear variability (the mean test score for a prior year was 66). The changing demographics of our students could also play a part, since progressively fewer first-year 


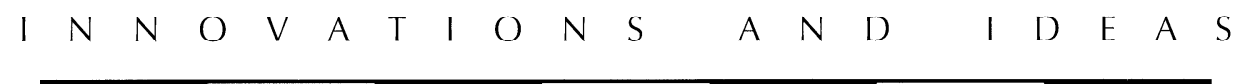

TABLE 1

Student exam scores before and after incorporation of case study analysis into a two-semester course in human anatomy and physiology

\begin{tabular}{ccc}
\hline Exam No. & Before & After \\
\hline \multicolumn{3}{c}{ First semester } \\
1 & 72 & 74 \\
2 & 62 & 70 \\
3 & 68 & 76 \\
& Second semester \\
1 & 64 & 72 \\
2 & 64 & 72 \\
3 & 66 & 74 \\
Mean \pm SE & $66 \pm 1.5$ & $73 \pm 0.9$ \\
\hline
\end{tabular}

There were 50 multiple choice questions in each exam. Scores reflect percent answered correctly. Values before incorporation of case study analysis represent data obtained during 1993-1994 academic year. Values after incorporation of case study analysis represent data obtained during 1994-1995 academic year.

students and more second- or third-year students have enrolled in our course in recent years. We had also increased the class time from 2 to $3 \mathrm{~h} / \mathrm{wk}$ to accommodate the case work. Nevertheless, it is likely that the introduction of case studies made a substantial contribution to the improvement in our class exam scores.

This conclusion is supported by the survey results in which most of our students indicated that their work on the case studies made the subject material easier to learn and helped them solidify their understanding of anatomy and physiology. The benefits of the case study analysis as perceived by the students are shown by a measurable improvement in the student exam performance.

\section{DISCUSSION}

Human anatomy and physiology is a content-driven course of study. Is the method of directed case analysis an appropriate means for helping undergraduates to master it? Herreid (2) alludes to two approaches for using case analysis in science classes. In the first, case analysis becomes the primary means of student learning. Can a prominent or even exclusive use of case studies be an effective way to teach human anatomy and physiology, particularly to lower-division nonscience (i.e., allied health, nursing, education) students? Some medical schools have successfully made use of case analyses in their problembased learning approach to teaching, even with students in the initial years of their basic science training $(2,8)$. In theory, this method could also work with undergraduates. We are not aware, however, of any program that is successfully doing so. Furthermore, we foresee that the intellectual inexperience and academic immaturity of lower-division students could make exclusive or primary reliance on case-based learning problematic. Morcover, as Herreid (2) suggests, "Even devotees [of the approach] admit that it is not the best method to deliver a plethora of facts, figures and principles."

Herreid (2) also suggests that the occasional use of case analyses can "spice up the semester and show students how their esoteric learning impacts on the world." This has been the approach taken by many anatomy and physiology instructors and by the authors of the major introductory textbooks in anatomy and physiology. The cases are given to provide realworld relevance or to enhance motivation for continued learning of the scientific facts and figures. Yet, the instructional utility of this case approach is open to question. Our experience with the cases found in the textbooks suggests that students tend to skip, ignore, or casually read them if the instructor makes only occasional or limited use of them. Unless emphasized, this motivational use of cases appears to have little benefit in improving student understanding of the facts and concepts of anatomy and physiology.

A regular program of case analysis is a suitable compromise. Students work the cases frequently enough to become accustomed to learning from them. Enough weight is placed on the grading of case analyses so that each student sees the importance of successfully solving them. Yet the cases remain learning accessories. They are not intended to be the primary means of instruction. Lectures, textbook reading, and laboratory exercises still serve as the most important methods for conveying the vast amount of facts, figures, and principles that a student must learn.

When used in such a fashion, we have found the directed case approach to be a highly valuable learning accessory. When used regularly, it reinforced facts and concepts and helped solidify student understanding in ways that few other methods were able. Case analysis promoted active learning. Students were forced to read their textbooks and supplemental materials to find the solutions to their cases, rather than relying passively on the instructor to provide the 
answers. They had to analyze appropriate graphs, figures, and tables. Complete responsibility for learning a particular subject was passed on to the student if the instructor chose to assign a case on textual material that was not covered in the lecture. As they solved the cascs, students were forced to reformulate concepts in their own words, integrate diverse principles in anatomy and physiology, and decide what information is important and what is superfluous. In this way, students were also improving their higherorder reasoning skills. Finally, the cases regularly reminded students that what they are learning about the human body has relevance in the real world of medicine, health, and fitness.

Our experience with using directed case analysis in an integrated course in anatomy and physiology has convinced us that case studies can be a helpful adjunct. The students have indicated their enthusiasm for working the case studies, and they have brought measurable improvement in student comprchension of the subject material (as measured by exam performance). They represent an extra avenue for teaching the content of human anatomy and physiology that complements the traditional approach of lecture, textbook, and laboratory.

\section{APPENDIX I: GUIDELINES FOR CASE STUDY ANALYSIS}

This semester, we will be analyzing "cases in human anatomy and physiology." Most simply, cases are stories-real or simulated-that illustrate important features of a field of study. In this course, we will analyze a selection of cases that illuminate important concepts of human anatomy and physiology. $\Lambda$ case study may involve a case bistory (the description of the symptoms and the progression of a medical disorder), but it doesn't have to. What is essential is that we address a real-world concern, i.e., a question, situation, or problem, by using the knowledge that we have gained about human anatomy and physiology.

\section{Issues to Keep in Mind in Approaching Cases}

In approaching the cases, you must keep the following issues in mind. What specific types of cells, tissues, organs, or organ systems are involved? What is the normal anatomy?
How do these structures work normally? What is the normal physiology?

What question must be answered? What problem must be solved? What specific situation must be addressed? What anatomical structures may be working abnormally? What are the physiological problems?

On the basis of your interpretation of the situation or problem, what medical or biological solution(s) could be proposed?

\section{Suggestions for Successfully Answering the Case Study Questions}

To answer the case study questions correctly, you must read them carefully and consider exactly what is asked for. The importance of your knowing exactly what a question is asking cannot be overstressed. Ask an instructor if you'rc unsure.

DON'T necessarily think that simply copying a long section of relevant information from the text provides a suitable answer. You must ANSWER the QUESTION, NOT merely find facts.

If you QUOTE or PARAPHRASE the textbook you must give a page reference. Similarly, you must make proper reference to the lecture notes.

Imaginative speculation. Imaginitive speculation, where appropriate, is encouraged (and will be rewarded), i.e., "It is possible that a drop in plasma levels of calcium under these conditions would make the hormone imbalance worse since..."

Brevity. Brevity will be rewarded. An answer in two sentences that can be provided in one should be. As Winnie-the-Pooh states, "I am a bear of very little brain and long words bother me."

When a question asks:

"Name..." This requires the name of something

"Name the top layer of the epidermis"

Answer: Stratum corneum. 


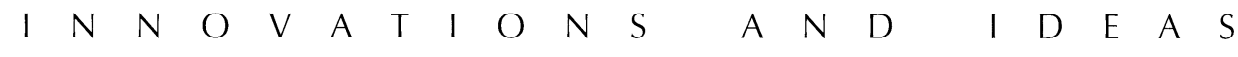

"List..." This requires a list of ALL of the items requested.

"List the major types of blood vessels."

Answer: Arteries, arterioles, capillaries, venules, veins.

"Explain..." This requires YOU to provide an explanation in YOUR OWN WORDS (not merely copying the text) of what is being asked?

"Explain the difference between an ion channel and a pump."

Answer: An ion channel is a membrane protein that permits ions to passively move across the cell membrane from regions of high concentration to regions of low concentration. A pump is a membrane protein that actively transports substances across the cell membrane from regions of low concentration to high concentration.

"What is..." This requires you to identify (or give a measurement of) the ITEM.

"What is the normal function of the heart?"

Answer: To pump blood.

"What is the approximate volume of blood in circulation?"

Answer: 5 liters.

"What is the role of voltage-gated potassium channels in producing the action potential?"

Answer: The opening of the voltage-gated potassium channels causes the repolarization and hyperpolarization of the membrane potential at the end of the action potential.

"How..." This requires you to describe a process, a mechanism, or a series of cause-and-effect events whereby something occurs.

"How does ATP use by skeletal muscles lead to contraction?",
Answer: ATP binds to the myosin cross bridge formed between the thick and thin filaments and causes the myosin head to be released from its active binding site on actin. The hydrolysis of ATP then causes the myosin head to be reoriented and reactivated (reenergized) in preparation for its reattachment to another actin molecule, swiveling of the myosin head, and the exertion of the force of contraction.

"Propose..." This requires you to present a logical coherent series of steps/actions that would SOLVE the problem as presented.

"Propose a way to treat cancer that relies upon what you know about the causes of uncontrolled growth of cells."

Answer: Because uncontrolled growth in many cancer cells is associated with abnormalities in protooncogenes and tumor suppressor genes, it would be reasonable to intervene in the activities of these genes or the proteins that are made from them. For cancers arising from defects in protooncogenes, it would be useful to pharmacologically inhibit the actions of the growth-promoting oncogenes once they are produced or to enhance the counterbalancing actions of existing tumor-suppressor genes. Conversely, for cancers arising from a loss of tumor-suppressor gene activity, it would be useful to also suppress the actions of protooncogenes or to restore the normal actions of the tumor suppressor genes by gene therapy or by giving pharmacological agents that mimic the actions of the tumor suppressor proteins that have been lost.

\section{APPENDIX II: REPRESENTATIVE CASE STUDY}

\section{Immune System Learning Objectives}

1) Understand the physiological mechanisms of an allergic reaction.

2) Recognize the symptoms of anaphylaxis and know the physiological condition necessary to induce it.

3) Identify and assess the different means of physiological exposure to antigens.

4) Identify the cellular steps in the immunology of allergen (antigen) sensitization. 
5) Know the process of inflammation, how it is induced, what chemical signals mediate it, and what alterations in the function of blood vessels occur.

6) Understand how local alterations in vascular function lead to general changes in the cardiovascular system/body fluid balance.

7) Understand the effects of sympathetic innervation/ catecholamine release on the cardiovascular/respiratory system.

\section{A Bad Day in the Park: Case Study on the Immune System}

A 31-year-old male is rushed from a local state park to the emergency room. The skin on his face, limbs, and torso is markedly swollen. He is having trouble swallowing and his breathing is labored and difficult. He feels faint, and a blood pressure determination reveals a systolic/diastolic pressure of $90 / 60 \mathrm{mmHg}$. His friends report that he had eaten a large picnic lunch earlier in the day. Later, he had taken a walk through a wildflower garden that was fully in bloom. Wandering off a path and into the woods, he had disturbed a wasp's nest. After being stung, he had to run through a large patch of poison ivy to escape from the wasps. Returning quickly to his friends, he rapidly developed the symptoms described above and was rushed to the hospital.

I) (1 pt) What is the name for the patient's allergic reaction?

Answer: Anaphylaxis (Ref. 5, p. 744).

2) (1 pt) List all of the potential allergens that he encountered at the park.

Answer: Food, plant pollen, wasp venom, chemical irritants in poison ivy.

3) (0.5 pt) What must happen to an allergen in order for it to induce such a reaction (i.e., where must it go once it enters the body)?

Answer: To induce anaphylaxis, the allergen must circulate systemically and affect mast cells throughout the body (Ref. 5, p. 744).
4) (2 pt) On the basis of this requirement, which of the allergens that you listed is most likely to have induced the reaction? Explain briefly. What types of allergic responses would you have expecled from the other allergens that you identified, and how would they have differed from the reaction observed?

Answer: Wasp venom. Because the wasp venom is injected into the capillary-rich tissue of the dermis of the skin, it is likely to be carried by the circulation (blood, lymph) to the rest of the body. Pollen in the air contacts the respiratory surfaces and is likely to induce a local type I response (hay fever; Ref. 5, p. 744 and 92). The chemical irritants in poison ivy diffuse into the skin from the surface and cause a delayed hypersensitivity in the immediate area of contact (Ref. 5, p. 92). Ingested food can be allergenic. The response is local and typically involves digestive disturbances upon contact. However, some foods have permeable antigens that can causc anaphylaxis.

5) (0.5 pt) Before this form of allergic reaction can occur what type of defensive cell has to have previously encountered the allergen to sensitize the individual?

Answer: Macrophages must engulf, process, and present the allergen (antigen; Ref. 5, p.745).

6) (0.5 pt) Which immune cell responds next in the process of sensitization to the allergen?

Answer: B cells (and T cells; Ref. 5, p. 745).

7) $(0.5 \mathrm{pt})$ What type of immunoglobulin docs this cell produce?

Answer: Cells produce immunoglobulin E (Ref. 5, p. 744-745).

8) (0.5 pt) What types of cells will then carry this antibody on their membranes in anticipation of a second exposure to the antigen?

Answer: Mast cells and basophils (Ref. 5, p. 744-745).

9) (0.5 pt) How do these cells respond when they encounter the antigen? What chemical signals do these cells release? 


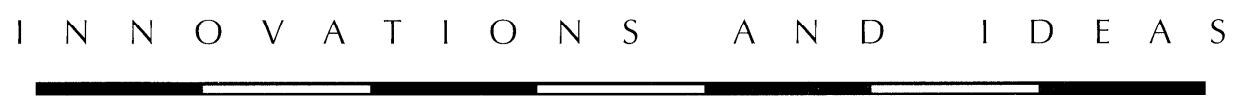

Answer: When antigens encounter the antibodies carried on the surface of these inflammatory cells, this induces the release of bistamine, beparin, monokines (Ref. 5, p. 744-745), kinins, and prostaglandins (class notes).

10) (1 pt) How does the release of these chemical signals lead to the pathological symptoms observed? List each of the symptoms and explain how the chemical signals would cause them.

Answer: Drop in blood pressure/faintness-caused by peripheral vasodilation (induced by inflammatory chemicals). Swelling of skin-caused by increased capillary permeability in skin (inflammatory chemicals). Difficulty swallowing (due to swelling of pharynx)-caused by increased capillary permeability in skin (inflammatory chemicals). Difficulty breathingconstriction of respiratory passageways (inflammatory chemicals; Ref. 5, p. 745).

11) (0.5 pt) Is this response an immediate or delayed bypersensitivity?

Answer: Immediate (type I; Ref. 5, p. 745 and Ref. 7a, p. 92).

12) (1.5 pt) Why would epinepbrine be a good treatment for the patient's condition, i.e., how would epinephrine help to alleviate each pathological symptom? Explain briefly.

Answer: Epinephrine (like sympathetic nervous stimulation) causes vasoconstriction in skin and digestive viscera (Ref. 5, Table 16-4); reduces blood flow to skin (increases blood pressure, reduces delivery of fluids to the skin, reduces swclling); reduccs blood flow to digestive viscera (Ref. 5, Table 16-1; reduces fluid delivery to tissues of pharynx, increase blood pressure); causes increased cardiac output (increased blood pressure); causes dilation of respiratory passageways (Ref. 5, Table 16-4; restores normal breathing); and causes inhibition of the release of inflammatory chemicals (3).

We thank Clyde Herreid and Nancy Cliff for critical reading of the manuscript and Connie Odrzywolski for secretarial assistance.

This material was presented at the Case Studies in Science Workshop sponsored by the Office of Teaching Effectiveness, State University of New York at Buffalo, May 31-June 2, 1995.

Address for reprint requests: W. H. C.liff, Dept. of Biology, Niagara University, Niagara, NY 14109 (E-mail: BCLIFF@Eagle.niagara.edu).

Received 26 June 1995; accepted in final form 7 February 1996.

\section{References}

1. Berne, R. M., and M. N. Levy. Case Studies in Physiology (3rd ed.). St. Louis, MO: Mosby, 1994.

2. Herreid, C. F. Case studies in science. A novel method of science education. J. Coll. Sci. Teach. 23: 221-229, 1994

3. Lichtenstein, L. Allergy and the immune system. Sci. Am. 269: 116-124, 1993.

4. Marieb, E. N. Human Anatomy and Physiology (3rd ed.). Redwood City, CA: Benjamin/Cunnings, 1995

5. Martini, F. Fundamentals of Anatomy and Physiology (2nd. ed.). Englewood Cliffs, NJ: Prentice-Hall, 1992

6. Martini, F. Fundamentals of Anatomy and Physiology (3rd ed.). Englewood Cliffs, NJ: Prentice-Hall, 1995.

7a.Martini, F., W. C. Ober, C. F. Garrison, and K. Welch. Clinical Manual for Fundamentals of Analomy and Pbysiol$o g y$ (2nd ed.). Englewood Cliffs, NJ: Prentice-Hall, 1992.

7. Martini, F., K. Welch, and M. Newsome. Applications Manual for Fundamentals of Anatomy and Physiology (3rd ed.). Englewood Cliffs, NJ: Prentice-Hall, 1995.

8. Reagan, C. R., and R. P. Menninger. Ten years of basic medical physiology in the Mercer problem-based curriculum. Am. J. Physiol. 266 (Adv. Physiol. Educ. 11): S24-S32, 1994.

9. Van Wynsherghe, D., and G. M. Colley. Case Histories in Human Physiology (2nd ed.). Dubuque, IA: Brown, 1996.

10. Van Wynsberghe, D., C. R. Noback, and R. Carola. Human Anatomy and Physiology (3rd ed.). New York, NY: McGrawHill, 1995. 